\title{
Micromicetos anemófilos de patogenicidad condicionada y biodeterioradores, en ambientes interiores del Centro Cultural de San Marcos
}

Carmen Rosa Méndez-Farro, Vilma Ruth Béjar-Castillo, German Vergaray-Ulffe, Hilda Yolanda Morante-Oliva, Roger Aníbal Gamboa-Ruiz, Jaqueline Getrudis Soberon-Amado

Facultad de Ciencias Biológicas, Universidad Nacional Mayor de San Marcos, Lima, Perú

Recibido el 15 de noviembre del 2018. Aceptado el 21 de noviembre del 2018.

DOI: https://doi.org/10.33017/RevECIPeru2018.0011/

\section{Resumen}

El presente estudio tiene como objetivo aislar e identificar micromicetos anemófilos potencialmente patógenos para el humano y posibles biodeterioradores de bienes del Centro Cultural de San Marcos. Se seleccionaron dos ambientes interiores, el depósito de retratos (DR) y el depósito de arte contemporáneo (DAC); se tomaron 48 muestras, 24 en cada ambiente, 6 en cada estación, 3 a las 11.00 h y 3 a las 15.00 h. Para la toma de muestra se empleó el método gravimétrico; para la identificación se hicieron cultivos y se utilizó la técnica de microcultivo en lámina. Se aislaron 96 cepas de micromicetos anemófilos correspondientes a 12 géneros. El $95,83 \%$ (92) fueron mohos y el 4,17\% (04) levaduras; el género más frecuente fue Penicillium $60,42 \%$ seguido de Cladosporium 18,75\%. La mayor frecuencia de aislamientos se produjo en verano $45,83 \%$. El ambiente con mayor frecuencia de aislamientos fue el depósito de arte contemporáneo 63,79\%. Se demostró la presencia de micromicetos pertenecientes a los géneros Cladosporium, Aspergillus y Paecilomyces asociados a enfermedades en el humano; y de Penicillium y Cladosporium asociados al deterioro de bienes de origen orgánico.

Descriptores: Micromicetos, Aire interior, Contaminación, Patogenicidad, Deterioro

\section{Abstract}

The objective of this study is to isolate and identify potentially pathogenic anemophilic micromycetes for humans and possible biodeteriorators of goods from the San Marcos Cultural Center. Two interior environments were selected, the portrait deposit (DR) and the contemporary art deposit (DAC); 48 samples were taken, 24 in each room, 6 in each station, 3 at $11.00 \mathrm{~h}$ and 3 at $15.00 \mathrm{~h}$. The gravimetric method was used to collect the sample; for identification, cultures were made and the microculture technique was used in foil. 96 strains of anemophilic micromycetes corresponding to 12 genera were isolated. 95,83 \% (92) were molds and 4,17\% (04) were yeasts; the most frequent genus was Penicillium $60,42 \%$ followed by Cladosporium 18,75\%. The highest frequency of isolations occurred in summer $45,83 \%$. The environment with the highest frequency of isolations was the deposit of contemporary art $63,79 \%$. The presence of micromycetes belonging to the genera Cladosporium, Aspergillus and Paecilomyces associated with diseases in humans was demonstrated; and of Penicillium and Cladosporium associated with the deterioration of goods of organic origin.

Keywords: Micromycetes, Indoor air, Contamination, Pathogenicity, Deterioration 


\section{Introducción}

Los micromicetos no habitan en el aire, pero a través de él se diseminan muchas de sus especies, a ellos se les denomina micromicetos anemófilos. Su transporte que también incluye a sus metabolitos se puede realizar a través de las corrientes de aire, partículas de polvo, gotas de agua, gotas de saliva, fragmento de hojas secas, animales, humanos, etc.

El aire es un ambiente hostil para los micromicetos, especialmente por la escasez de nutrientes, sin embargo, suele contener gran cantidad de ellos [1, 2 , 3]. Las estructuras especialmente adaptadas para su diseminación son las esporas y conidios, pero cualquier fragmento del micromiceto puede dar origen a una nueva colonia, esta característica favorece el aumento de la concentración de micromicetos viables en el aire.

Los micromicetos anemófilos, que pueden ser filamentosos o levaduriformes, son cosmopolitas, se encuentran ampliamente distribuidos en toda la biosfera, generalmente desarrollan sobre materia orgánica muerta, aunque pueden ser parásitos 0 comensales de humanos, animales o plantas causando grandes perjuicios a los seres vivos. Cuando crecen en los ambientes interiores pueden desarrollar sobre cualquier sustrato orgánico o que contiene polvillo con componentes orgánicos.

Los seres humanos pasan la mayor parte de su tiempo en ambientes interiores como viviendas, escuelas, universidades, orfanatos, hospitales, lugares de trabajo, lugares de entretenimiento, restaurantes, medios de transporte, etc. Los niveles de contaminación por micromicetos en el aire de dichos ambientes pueden ser de 2 a 100 veces más elevados que en el aire exterior [4], lo cual aumenta el riesgo para la salud de los humanos y para el mantenimiento de sus bienes.

Los micromicetos o partículas de ellos que están en el aire, al ser inhalados o entrar en contacto con la piel y mucosas ocasionan con frecuencia, en personas sensibles, problemas respiratorios de hipersensibilidad, como rinitis y asma bronquial; también conjuntivitis y urticaria; y en personas con deficiencia inmune pueden producir infecciones severas como aspergilosis y criptococosis $[5,6,7]$. Los grupos etáreos más afectados son los niños, adolescentes y adultos jóvenes [8, 9].

Condiciones ambientales tales como temperatura, humedad relativa, corrientes de aire, sustratos de origen orgánico, suciedad, polvillo orgánico y presencia de animales y humanos influyen de manera determinante en la proliferación y diseminación de fragmentos de micromicetos [10, 11].

Según la Agencia para la Protección del Medio Ambiente de los Estados Unidos de Norteamérica [12], los contaminantes del aire interior están entre los cinco mayores riesgos para la salud pública.

Los micromicetos también deterioran bienes útiles como: alimentos, ropa, libros, documentos, muebles, obras de arte, piezas de museo, etc. [8]. $\mathrm{Se}$ han efectuado numerosos estudios con la finalidad de demostrar la presencia de micromicetos en ambientes interiores; los que se han aislado con mayor frecuencia son los filamentosos Cladosporium, Penicillium, Aspergillus, Fusarium, Alternaria y Acremonium; y las levaduras Rhodotorula y Candida [13, 14, 15].

El Centro Cultural de la Universidad Nacional Mayor de San Marcos, es un edificio del Centro Histórico de Lima, reconocido como patrimonio cultural de la humanidad que alberga museos, biblioteca y otras entidades culturales. Actualmente se carece de información sobre la presencia y diversidad de micromicetos anemófilos en sus ambientes interiores, por lo que se ignora los riesgos para la salud de sus trabajadores y del público asistente; y la posibilidad de deterioro de documentos, libros, lienzos, mobiliario y otros bienes que constituyen parte del patrimonio cultural del Perú.

Por lo expuesto, el objetivo del estudio es aislar e identificar los micromicetos anemófilos que se encuentran en dicho Centro Cultural y determinar aquellos que son potencialmente patógenos para el humano y aquellos que están relacionados con el deterioro de sus bienes.

\section{Metodología}

El estudio es de tipo descriptivo, transversal y observacional.

Se seleccionaron dos ambientes interiores del Centro Cultural de la Universidad Nacional Mayor de San Marcos, el Depósito de Retratos (DR) y el Depósito de Arte Contemporáneo (DAC); para ello se tomó como base la presencia de bienes culturales susceptibles de deterioro, polvillo, sustratos de origen orgánico, suciedad, humedad relativa, falta de ventilación y olor a moho. Se tomaron 48 muestras de aire, 24 en cada ambiente, 6 en cada estación del año, 3 a las 11.00 h. y 3 a las 
$15.00 \mathrm{~h}$. Los puntos de muestreo se repitieron en cada estación.

Para la toma de muestra se empleó el método gravimétrico de sedimentación en placa para muestreos ambientales propuesto por Omeliansky [3], se utilizaron placas de petri con agar papa dextrosa a las cuales se les agregó cloranfenicol $(0.1 \%)$.

Las placas se ubicaron a $1.50 \mathrm{~m}$ del suelo y se expusieron durante 15 minutos, luego se incubaron a $25{ }^{\circ} \mathrm{C}$ durante 5 días, al cabo de los cuales se contaron las colonias.

Posteriormente se procedió a la identificación de los micromicetos filamentosos, mediante la observación macroscópica de las características morfológicas de la colonia y microscópica de las estructuras de reproducción. Cuando no fue posible la identificación se procedió al aislamiento de la cepa y a la obtención del cultivo puro, mediante la técnica de dilución; y al microcultivo en lámina.

En los casos de levaduras, la lectura se realizó a las $48 \mathrm{~h}$ de incubación; las cepas aisladas se sembraron en caldo sabouraud glucosado (observación microscópica), agar sabouraud glucosado (observación macroscópica de la colonia), suero humano (tubo germinal), y agar almidón arroz (clamidosporas, pseudomicelio y blastosporas); como pruebas bioquímicas se realizaron ureasa en agar urea de Christensen y asimilación de los carbohidratos glucosa, sacarosa, maltosa, lactosa e inositol en agar Hugh-Leifson.

En cada ambiente paralelamente al muestreo se midió la temperatura y la humedad relativa utilizando el Wired thermometer/ hygrometer 631032 radio shack.

\section{Resultados y Discusión}

Se aislaron 96 colonias de micromicetos anemófilos, de los cuales el 95,83 \% (92) fueron filamentosos y el 4,17\% (04) levaduras.

Los micromicetos aislados pertenecen a 12 géneros, los de mayor frecuencia fueron Penicillium 60,42 \% (58), Cladosporium 18,75\% (18), Candida $4,17 \%$ (04) y Aspergillus 3,13\% (03).

Los géneros de micromicetos aislados son similares a los reportados en estudios realizados en ambientes interiores en otros países; la diferencia está en la mayor frecuencia de determinados géneros $[1,3,13,16]$.

En verano se aisló el mayor número de colonias $45,83 \%$ (44) del total, seguido de primavera 27,08 $\%$ (26); en verano y primavera el micromiceto más frecuente fue Penicillium con el 68,18\% (30) y el $73,08 \%$ (19) respectivamente.

En invierno, se aisló el menor número de colonias $12,50 \%$ (12), los micromicetos más frecuentes fueron Penicillium y Cladosporium ambos con el 25 $\%$ (03) de las colonias. Tabla 1.

En la estación de verano la temperatura promedio en los ambientes estudiados fue de $27,20^{\circ} \mathrm{C}$ y la humedad relativa de $70,5 \%$; y en la de invierno de $18,65^{\circ} \mathrm{C}$ y $67,65 \%$ respectivamente.

En las estaciones de verano, otoño y primavera los valores de temperatura y humedad relativa en los dos ambientes evaluados estuvieron por encima de los valores sugeridos para controlar la contaminación en ambientes internos, que son 21 ${ }^{\circ} \mathrm{C}$ y $50 \%$ respectivamente [17]. En invierno, la temperatura promedio fue notablemente inferior a las otras estaciones, en cambio la humedad relativa promedio fue similar; este dato evidencia que la temperatura fue el factor que influyó en la mayor frecuencia de aislamientos, criterio diferente al enunciado por Villafañe et al [18] quienes concluyen que en Cartagena de Indias (Colombia) no se pudo determinar la influencia de la temperatura.

De los ambientes, el mayor número de micromicetos aislados se obtuvo en el depósito de arte contemporáneo $56,25 \%$ (54) y en la tarde $63,79 \%$; posiblemente debido al mayor número de piezas de museo que presentan mayor extensión de superficies, al mayor polvillo existente y a la mayor circulación de personas.

Los análisis efectuados a las levaduras aisladas revelan que pertenecen al género Candida pero no a la especie Candida albicans.

Entre los micromicetos aislados hay algunos géneros que están relacionados con enfermedades, entre ellos Cladosporium que está asociado a cuadros de hipersensibilidad, en particular a enfermedades respiratorias alérgicas, también se le ha incriminado en casos de cromoblastomicosis y cromomicosis cerebral; Paecilomyces que está involucrado en casos de endocarditis, sinusitus maxilar crónica, queratitis micótica y endoftalmitis; Aspergillus cuyas manifestaciones más comunes 
son: infección broncopulmonar alérgica, aspergiloma y neumonía invasiva, también está asociado con cuadros de micotoxicosis. La levadura Candida también puede afectar la salud del humano. Por otro lado, los géneros Cladosporium y
Penicillium están relacionados con el deterioro de libros, pinturas, pieles, maderas, piezas de museo, etc. $[5,7,8,9]$.

Tabla 1. Frecuencia de micromicetos por estación y por recinto

\begin{tabular}{|c|c|c|c|c|c|c|c|}
\hline \multirow{3}{*}{ Estación } & \multirow{3}{*}{ Micromiceto } & \multicolumn{4}{|c|}{ Recinto } & \multirow{2}{*}{\multicolumn{2}{|c|}{ Total }} \\
\hline & & & DR & & DAC & & \\
\hline & & № & $\%$ & № & $\%$ & № & $\%$ \\
\hline \multirow{9}{*}{ Verano } & Penicillium & 14 & 66.67 & 16 & 69.57 & 30 & \\
\hline & Cladosporium & 2 & 9.52 & 6 & 26.09 & 8 & \\
\hline & Aspergillus & 2 & 9.52 & 0 & 0 & 2 & \\
\hline & Hormodendrum & 0 & 0 & 1 & 4.35 & 1 & \\
\hline & Rhizopus & 1 & 4.76 & 0 & 0 & 1 & \\
\hline & Geotrichum & 0 & 0 & 0 & 0 & 0 & \\
\hline & Nigrospora & 1 & 4.76 & 0 & 0 & 1 & \\
\hline & Candida & 1 & 4.76 & 0 & 0 & 1 & \\
\hline & Subtotal & 21 & 100 & 23 & 100 & 44 & 45.83 \\
\hline \multirow{5}{*}{ Otoño } & Penicillium & 2 & 50 & 4 & 40 & 6 & \\
\hline & Cladosporium & 1 & 25 & 5 & 50 & 6 & \\
\hline & Nigrospora & 0 & 0 & 1 & 10 & 1 & \\
\hline & Candida & 1 & 25 & 0 & 0 & 1 & \\
\hline & Subtotal & 4 & 100 & 10 & 100 & 14 & 14.58 \\
\hline \multirow{8}{*}{ Invierno } & Penicillium & 1 & 25 & 2 & 25 & 3 & \\
\hline & Cladosporium & 1 & 25 & 2 & 25 & 3 & \\
\hline & Aspergillus & 0 & 0 & 1 & 12.5 & 1 & \\
\hline & Geotrichum & 1 & 25 & 1 & 12.5 & 2 & \\
\hline & Cephalosporium & 0 & 0 & 1 & 12.5 & 1 & \\
\hline & Acremonium & 1 & 25 & 0 & 0 & 1 & \\
\hline & Candida & 0 & 0 & 1 & 12.5 & 1 & \\
\hline & Subtotal & 4 & 100 & 8 & 100 & 12 & 12.50 \\
\hline \multirow{7}{*}{ Primavera } & Penicillium & 8 & 61.54 & 11 & 84.62 & 19 & \\
\hline & Cladosporium & 1 & 7.69 & 0 & 0 & 1 & \\
\hline & Paecilomyces & 3 & 23.08 & 0 & 0 & 3 & \\
\hline & Trichoderma & 1 & 7.69 & 0 & 0 & 1 & \\
\hline & Hormodendrum & 0 & 0 & 1 & 7.69 & 1 & \\
\hline & Candida & 0 & 0 & 1 & 7.69 & 1 & \\
\hline & Subtotal & 13 & 100 & 13 & 100 & 26 & 27.08 \\
\hline \multicolumn{2}{|l|}{ Total } & 42 & 43.75 & 54 & 56.25 & 96 & 100 \\
\hline
\end{tabular}

\section{Conclusiones}

En el Depósito de Retratos y en el Depósito de Arte Contemporáneo del Centro Cultural de la Universidad Nacional Mayor de San Marcos se aislaron e identificaron numerosas colonias de micromicetos anemófilos.

Se comprobó que la mayor temperatura, característica de los meses de verano, favorece su presencia y diseminación.
Los micromicetos aislados pertenecen a 12 géneros, algunos de los cuales como Cladosporium, Paecilomyces y Aspergillus están asociados a infecciones oportunistas en humanos y otros como Cladosporium y Penicillium están asociados al deterioro de maderas, libros, pinturas, lienzos y piezas de museo. 


\section{Agradecimientos}

A Fundación San Marcos para el Desarrollo de la Ciencia y la Cultura por el soporte económico brindado.

\section{Referencias}

[1] D. Hernandez-Velandia y L. LizarazoForero. Determinación y comparación aerobiológica en tres archivos de la empresa de energía de Boyacá, Tunja (Colombia). Salud Uninorte, Barranquilla (Col). 31 (2015) 537-547.

[2] P. Esquivel, M. Mangiaterra, G, Giusiano y MA. Sosa. Microhongos anemofilos en ambientes abiertos de dos ciudades del Nordeste Argentino. Bol. Micol. 18 (2003) 21-28.

[3] S. Fekadu y A. Melaku. Microbiological Quality of indoor air in University Libraries. Asian. Pac Trop Biomedic 1 (2014) S312S317.

[4] M.C. De la Rosa, M.A. Mosso y C. Ullán. El aire: hábitat y medio de transmisión de microorganismos. Observatorio medioambiental 5 (2002) 375-402.

[5] A. Díaz, D. Fabre, G. Countin y T. Gonzales. La sensibilización a hongos ambientales y su relación con enfermedades atópicas en escolares. Revista Cubana de Medicina General Integral 26 (2010) 647-655.

[6] M. S. Perzanowski et al. Association of sensitization to Alternaria allergens with asthma among school-age children. J Allergy Clin. Inmunol 101 (1998) 626-632.

[7] J. Ponton et al. Hongos y actinomicetos alergénicos. Rev Iberoam Micol 1 (2002) 151.

[8] V. Sánchez. Estudio aerobiológico de las esporas fúngicas en interiores, influencia sobre la salud y procesos de biodeterioro. Tesis de fin de Grado, Universidad de JaénEspaña. 2016.

[9] B. Brunekreef, DW. Dockery and M. Krzyzanowski. Epidemiologic studies on short-term effects of low levels of major ambient air pollution components. Environ Health Perspect. 103 (1995) 3-13.
[10] P. Fsadni, F. Bezzina, C. Fsadni, S. Montefort. The Impact of Microbiological Pollutants on School Indoor Air Quality. Journal of Geoscience and Environment Protection 5 (2017) 54-65. doi: 10.4236/gep.2017.55004.

[11] M. Huang, L. Guan, L. Zhoud y D. Ling. An Investigation of precipitation of airbone fungi particles in the rooms of university library. Human I Ko ta Hsueh Pao 22 (1997) 494-496.

[12] EPA Environmental Protection Agency: IAQ Reference Guide. Indoor air quality Tools for Schools. EPA 402-K-95-001-Third Edition. 2005.

[13] D. Bueno, J. Silva y G. Oliver. Hongos ambientales en una biblioteca: Un año de estudio. Anales de Documentación 6 (2003) 27-34.

[14] D.L. Toloza y L.M. Lizarazo. Calidad microbiológica del ambiente de la biblioteca Alfonso Patiño Rosselli, Tunja Boyacá Colombia. rev.udcaactual.divulg.cient. [online]. 16 (2013) 43-52.

[15] J. Torres et al. Aeromicrobiota de la biblioteca de la escuela de Bioanálisis "Prof. José M. Forero", Universidad Central de Venezuela. Academia Biomédica Digital. 64 (2015) 1-12.

[16] T. Alonso et al. Aislamiento de hongos en instalaciones deportivas de la UNAM. Rev Fac Med UNAM 46 (2003) 93-96.

[17] S. Ogden. El Manual de Preservación de Bibliotecas y Archivos del Northeast Document Conservation Center. (Biblioteca Nacional de Venezuela, 1998) Fascículo dos. p. 5-18

[18] L. Villafañe et al. Determinación de la carga fúngica anemófila en seis sectores de la ciudad de Cartagena de Indias. Ciencia y

Email: Salud Virtual 1 (2009) 15-20.

cmendezf08@hotmail.com

vilmabejar@hotmail.com

germanvergaray@gmail.com

hmoranteo@hotmail.com

roggeiro17@hotmail.com

jaqueline.soberon@hotmail.com 